A N N A L E S Annales de Bretagne et des Pays de l'Ouest

\title{
Richard E. Barton, Lordship in the county of Maine, C. $890-1160$
}

\section{Florian Mazel}

\section{(2) OpenEdition}

Édition électronique

URL : http://journals.openedition.org/abpo/844

DOI : $10.4000 / a b p o .844$

ISBN : 978-2-7535-1502-4

ISSN : 2108-6443

\section{Éditeur}

Presses universitaires de Rennes

Édition imprimée

Date de publication : 30 juin 2006

Pagination : 185-188

ISBN : 978-2-7535-0331-1

ISSN : 0399-0826

\section{Référence électronique}

Florian Mazel, «Richard E. Barton, Lordship in the county of Maine, c. 890-1760», Annales de Bretagne et des Pays de l'Ouest [En ligne], 113-2 | 2006, mis en ligne le 30 juin 2008, consulté le 23 septembre 2020. URL : http://journals.openedition.org/abpo/844 ; DOI : https://doi.org/10.4000/abpo.844 


\section{Comptes rendus}

BARTON, Richard E., Lordship in the county of Maine, c. 890-1160, The Boydell Press, Woodbridge, 2004, 255 p., index, cartes et tableaux généalogiques.

L'ouvrage de Richard Barton constitue la version allégée de sa thèse de doctorat, soutenue devant l'Université de Santa Barbara (Californie), hélas amputée par l'éditeur de ses annexes documentaires. Il faut d'abord souligner quel grand plaisir procure le fait de voir l'obscur passé de la vieille Europe continuer de susciter outre-Atlantique l'intérêt de chercheurs passionnés et souvent talentueux, combien aussi les questionnements qui les animent apportent un vent d'air frais dans nos débats franco-français ou européens; et cela d'autant plus que R. Barton a l'élégance de choisir une échelle et un genre historiographique, la monographie régionale - ici l'ancien comté du Maine -, typiquement français et fort peu à l'honneur en Amérique. À ce titre et sur bien des sujets qu'il aborde, le propos de R. Barton, tout en apportant son lot de solides mises au point érudites, apparaît revigorant. Il n'en comporte pas moins, à nos yeux, un certain nombre de faiblesses et de facilités qui en altèrent l'efficacité.

Le livre est porteur d'une véritable thèse, celle de la forte continuité du dominium aristocratique, dans son contenu comme dans son exercice, entre la fin du IX et la fin du XII ${ }^{e}$ siècle, un problème qui demeure d'actualité en dépit de la lassitude et de la confusion qui règnent souvent aujourd'hui parmi les chercheurs français à ce sujet. L'auteur affiche de manière résolue et explicite un positionnement historiographique très marqué, qui le rattache au courant de l'anthropologie juridique anglo-saxonne et à certains de ses prolongements français, en l'occurrence les travaux de Dominique Barthélemy. Rejetant toute approche "institutionnelle ", il entend démontrer l'inutilité de la thèse d'une forte mutation socio-politique autour de l'an mil, en dépit de l'indéniable processus de décentralisation du pouvoir (du roi au duc des Francs, du duc au comte, du comte aux châtelains...) qui touche le Maine comme le reste du royaume de France. Parallèlement, il s'attache à souligner le primat des éléments " charismatiques " (le concept wébérien est ici laïcisé) de la seigneurie, à savoir le primat du prestige personnel, de l'approbation des pairs, des liens d'amitié, sur toutes les autres formes de liens politiques et sociaux (la vassalité, la parenté...). Le propos se déploie à travers huit chapitres : le premier définit les grands cadres politiques entre 850 et 975 ; le second étudie la rivalité entre le comte et l'évêque jusqu'au milieu du $\mathrm{xI}^{\mathrm{e}}$ siècle; les troisième et quatrième chapitres décrivent le pouvoir comtal (ses fondements matériels et symboliques, la question des relations de fidélité et de l'entourage du comte); le cinquième traite de l'articulation des dimensions " publique " et " privée " au sein de la seigneurie (la justice, les châteaux, les coutumes); les sixième et septième de la violence et des conflits; le huitième chapitre envisage enfin le problème de la féodalité stricto sensu. 
Sur de nombreux points le propos est convaincant. On peut ainsi retenir, dans la postérité des nombreux et récents travaux des spécialistes du haut Moyen Âge, une critique saine et argumentée de l'idéalisation dont l'époque carolingienne fait l'objet de la part de bien des historiens "mutationistes ", depuis Duby et Lemarignier, tant sur le plan de la justice et de l'administration publiques, que sur celui de la violence ou de la fidélité aristocratiques. Il est évident qu'en ce domaine le poids de la tradition jacobine dans l'historiographie française teinte souvent d'anachronisme les conceptions que l'on se fait, ici, du pouvoir " carolingien " ou "féodal ", à travers, par exemple, des oppositions schématiques (et souvent moralisantes) entre "public " et " privé ", " central " et " local ", " laïque " et " ecclésiastique". Les formes du pouvoir comtal aux Xe-Xle siècles sont aussi très finement analysées (domaines, titulature, monnaie, entourage...). Il en ressort en particulier la faiblesse de l'emprise comtale sur les abbatia (par ailleurs très peu nombreuses avant le renouveau de la fin $\mathrm{X}^{\mathrm{e}}$-début Xl ${ }^{\mathrm{e}}$ siècle) et l'importance déterminante, tout au long de la période considérée, du contrôle de la cité du Mans. Dans ce cadre, la rivalité avec l'évêque est à la fois une matrice et un sérieux handicap pour les comtes. À la différence des comtés voisins (Angers, Rennes, Nantes, Chartres...), l'episcopatus du Mans échappe au comte et dispose d'une puissance et d'une légitimité anciennes et redoutables, au point de servir d'appui, à partir du milieu du x $\mathrm{x}^{\mathrm{e}}$ siècle, à l'essor des familles aristocratiques locales qui parviennent à s'en emparer (les vicomtes du Maine d'abord, les sires de Bellême ensuite). Le comte du Maine apparaît ainsi dépourvu des honores ecclésiastiques alors si indispensables à la réussite princière. L'exaspération de la lutte entre le comte et l'évêque apparaît en outre avoir directement favorisé l'apparition des châteaux et des premières seigneuries castrales au tournant des $\mathrm{X}^{\mathrm{e}}$ et $\mathrm{XI}^{\mathrm{e}}$ siècles, nouveau facteur de l'affaiblissement comtal. Un autre aspect important de l'étude de R. Barton est de relever l'ancienneté de l'implantation d'une aristocratie locale bien souvent à l'origine des futurs " lignages châtelains ". Une famille comme celle des futurs vicomtes du Maine semble par exemple avoir joué un rôle décisif dans la victoire du comte Roger sur son concurrent Gauzlin à la fin du IX ${ }^{\mathrm{e}}$ siècle et donc dans l'implantation de la seconde dynastie des comtes du Maine (les Hugonides). Cette situation explique d'ailleurs un autre phénomène justement souligné par R. Barton, à savoir la non territorialisation des fidélités aristocratiques tout au long de la période, jusqu'à la construction Plantagenêt : ajuster fidélités nobiliaires, contours d'un comté et puissance d'une dynastie comtale n'a alors guère de sens. Remarquable encore est l'étude des "mauvaises coutumes ". Après avoir montré combien la distribution chronologique globale des mentions conservées ne s'accorde pas du tout à la séquence " mutationniste ", R. Barton décrit avec finesse les différents phénomènes qui expliquent le surgissement de l'expression sous la plume monastique, qu'il s'agisse de la redéfinition douloureuse des aires vicariales, de la revitalisation des anciennes immunités par les nouveaux établissements du monachisme réformé, de la diffusion d'une stratégie rhétorique de délégitimation de la seigneurie laïque, à rapprocher des formules de malédictions, ou encore de l'impact des bouleversements politiques et militaires (comme la poussée angevine en Saumurois et Touraine dans les années 1020-1050). On est loin de l'apparition de nouvelles formes de prélèvement et l'analyse de R. Barton pour le Maine rejoint ici celles de plusieurs autres historiens pour d'autres espaces (E. MagnouNortier, M. Bourin, C. Duhamel-Amado, Y. Sassier).

Au passage, R. Barton opère par ailleurs un certain nombre de révisions érudites le plus souvent justifiées. Contre O. Guillot et G. Louise, il montre que le siège du Mans échappe à l'emprise du duc des Francs comme à celle du roi dès 
l'accession de Mainard, en 951, et non vers 1040. Contre toute la tradition historiographique régionale, il soutient, de manière assez convaincante, que la restauration de l'abbaye de La Couture est le fruit de l'action conjuguée de l'évêque et de l'abbé de Saint-Julien de Tours vers 997-1004 et que les comtes ne s'intéressent vraiment à l'abbaye qu'avec Hélie de La Flèche (1091-1110), à la fin du $\mathrm{Xl}^{\mathrm{e}}$ siècle. Contre $\mathrm{B}$. Bachrach, il démontre que la poussée angevine au sud du Maine au Xl ${ }^{\text {e }}$ siècle n'est pas aussi générale et systématique qu'on l'avance et que, plus généralement, les fidélités aux différents princes (Anjou, Maine, Normandie, Bretagne) restent fragiles et multiples tout au long du siècle.

Cependant, en dépit de ses indéniables qualités, l'ouvrage suscite parfois un certain malaise, qui s'accentue à la lecture des trois derniers chapitres. Le propos présente d'abord un certain nombre de lacunes, que l'on ne s'explique guère et qui s'avèrent assez dommageables, me semble-t-il, à l'argumentation d'ensemble. Alors que les relations entre les comtes et l'Église figurent très justement au cœur de l'analyse du pouvoir comtal, on ne comprend pas pourquoi ces relations sont complètement négligées pour le reste de l'aristocratie. Certes, elles affleurent lorsque sont abordées la question des mauvaises coutumes ou celle du règlement des conflits, mais elles ne font jamais l'objet d'un traitement particulier. Quand on connaît, par exemple, l'importance des fondations de prieurés pour l'appropriation de la potestas et la revendication d'un statut seigneurial (le dominium), ou bien, plus matériellement, le rôle joué par ces fondations dans la genèse des pôles castraux, ou encore la part de la dîme au sein du prélèvement seigneurial, on peut s'étonner d'une telle " laïcisation " de la seigneurie. De même, alors que R. Barton reconnaît que la décentralisation du pouvoir a eu de notables conséquences sur les structures de parenté et le rapport à l'espace des groupes aristocratiques, il ne fait qu'évoquer ces phénomènes, sans les étudier ni même, apparemment, prendre conscience du fait qu'ils sont susceptibles de remettre en cause sa thèse d'une absence de transformation dans la nature même du pouvoir seigneurial. En réalité, peut-on cloisonner à ce point les différentes dimensions du pouvoir seigneurial et enclore une sorte de " cœur " dont seraient exclus, presque par principe, la dimension ecclésiale, la parenté, le rapport à l'espace, autant de domaines que l'historiographie récente, en particulier française et italienne, tend à considérer comme essentiels pour la compréhension de l'exercice mais aussi de la nature du dominium aux IX $\mathrm{e}^{\mathrm{e}}$ XII ${ }^{\mathrm{e}}$ siècles? À ce titre, les réserves dont font l'objet les études fondées sur l'anthropologie de la parenté ou les systèmes anthroponymiques, si elles peuvent se révéler justes au sujet de tel ou tel point d'érudition (ainsi pour le rôle de l'alliance entre les Hugonides et les Robertiens au début du $\mathrm{x}^{\mathrm{e}}$ siècle, sans doute exagéré par Karl Ferdinand Werner, ou les analyses onomastiques de Katherine Keats-Rohan au sujet des Rorgonides ou des seigneurs de Mayenne, excessivement péremptoires au regard de leurs fondements hypothétiques), ne peuvent légitimement déboucher sur la récusation implicite de ce type d'approche en général, surtout d'une manière aussi légère, qui relève plus de la position de principe que de la démonstration. D'autant qu'en la matière, R. Barton semble ignorer une bonne part de l'historiographie récente ${ }^{1}$. Un dernier aspect du livre suscite la gêne : lorsqu'il aborde la résolution des conflits et les liens féodo-vassaliques, l'auteur ne témoigne pas suffisamment, à nos yeux, d'un véritable sens

1. La bibliographie ne mentionne aucun des volumes de la grande enquête sur l'anthroponymie, coordonnée par Monique Bourin, publiés depuis 1990. De même, pour l'articulation des relations entre parenté et espace, on pourra s'étonner de l'absence de bien des travaux d'Anita Guerreau-Jalabert. 
de la critique historique, mais se contente d'appliquer de manière assez mécanique les modèles fourbis par l'anthropologie juridique d'une part, l'" anti-féodalité " d'E. Brown et $S$. Reynolds d'autre part, alors même que ces modèles, tout en ayant profondément dynamisé l'historiographie récente, peuvent faire l'objet de réserves ou pour le moins de sérieuses nuances ${ }^{2}$. En somme, les analyses de R. Barton trahissent souvent les mêmes excès méthodologiques et historiographiques, à front renversé, que bien des travaux de ses " adversaires " déclarés. Cela le conduit à des conclusions souvent simplificatrices, que révèle l'usage peu convaincant, au sujet des rapports sociaux et politiques, de termes ou d'expressions (peut-on parler de notions?) quelques peu filandreux, tels « fluidité ", " flexibilité ", " caractère informel "...

Au total, il s'agit donc d'un ouvrage utile, précieux même sur de nombreux points, mais qui souffre d'analyses parfois trop rapides et d'un certain emprisonnement dans les modes anglo-saxonnes. Cela dit sans acrimonie aucune de la part d'un lecteur et d'un chercheur qui sait reconnaître tout ce qu'il doit, personnellement, à cette historiographie vivifiante.

Florian MAZEL

AUTISSIER, Anne, La Sculpture romane en Bretagne, Xle-XIte siècles, Rennes, PUR, coll. "Art et société ", 2005, 380 p.

L'originalité de la sculpture romane bretonne tient, en premier lieu, à l'utilisation de matériaux a priori inappropriés : le grès et le granite. Les décors qui en sont issus peuvent nous apparaître frustes. Toutefois, au XII ${ }^{\mathrm{e}}$ siècle, les sculpteurs de la partie orientale de la Bretagne, ont été parfaitement capables de mâ̂triser ces matériaux pour y modeler des scènes similaires à celles des chapiteaux calcaires.

La sobriété de l'art roman se remarque tout d'abord dans les plans des églises qui étaient de dimensions modestes et de forme simple, en croix latine. Elle est également visible dans les élévations, souvent de type basilical. De grandes arcades à simple ou double rouleau percent les parois murales séparant la nef centrale et les collatéraux. La plupart des nefs des églises romanes bretonnes n'étaient sans doute pas voûtées de pierre, ce qui peut expliquer la fréquence de supports relativement simples. La sobriété transparaît aussi à l'extérieur avec des façades austères. Il y a peu de décorations. Cependant on ne peut nier l'harmonie des volumes.

2. Sur l'anti-féodalité, voir par exemple la note critique de Dominique Barthélemy à propos de l'ouvrage de S. Reynolds, Fiefs and vassals. The medieval evidence reinterpreted, Oxford, 1994 : "La théorie féodale à l'épreuve de l'anthropologie (note critique)", Annales HSS, mars-avril 1997, n 2, p. 321-341; ou la thèse d'H. Débax, La féodalité languedocienne, XP'-XII siècles. Serments, hommages et fiefs dans le Languedoc des Trencavel, Toulouse, 2003. Pour une critique de certains aspects de l'anthropologie juridique, et même s'il était bien évidemment impossible à R. Barton d'en avoir connaissance, on peut renvoyer aux différents articles publiés récemment par Bruno Lemesle, en attendant de disposer d'une publication de son mémoire d'habilitation sur Conflits, normes et justice dans la région angevine du milieu du XI à la fin du XII siècle (Université de Paris IV, 2005); ainsi qu'à F. Mazel, " Amitié et rupture de l'amitié. Moines et grands laïcs provençaux au temps de la crise grégorienne (milieu Xle-milieu XII ${ }^{\mathrm{e}}$ siècle) ", Revue historique, t. 307, 2005, p. 53-95. 